\title{
Impacto del sistema OLGA en la detección de gastritis crónica atrófica en Colombia: un estudio de casos y controles
}

\section{A Case and Control Study of the OLGA System's Impact on Detection of Chronic Atrophic Gastritis in Colombia}

Diana Martínez, MD, ${ }^{1}$ William Otero, MD, ${ }^{2}$ Orlando Ricaurte, MD. ${ }^{3}$

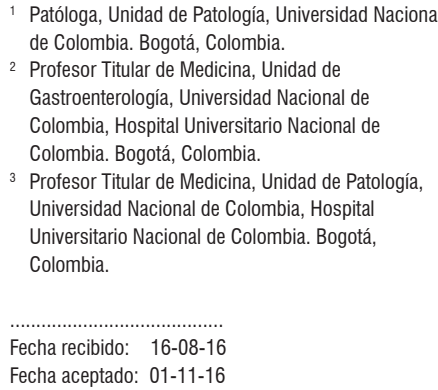

2 Profesor Titular de Medicina, Unidad de Gastroenterología, Universidad Nacional de Colombia, Hospital Universitario Nacional de Colombia. Bogotá, Colombia.

3 Profesor Titular de Medicina, Unidad de Patología, Universidad Nacional de Colombia, Hospital Universitario Nacional de Colombia. Bogotá, Colombia.

Fecha recibido: 16-08-16 Fecha aceptado: 01-11-16

\begin{abstract}
Resumen
Introducción: la gastritis crónica atrófica (GCA) es una entidad clinicopatológica relacionada con cáncer gástrico (CG) de tipo intestinal. Su principal causa es Helicobacter pylori. Actualmente, además del diagnóstico, se recomienda evaluar la extensión de la atrofia o de la metaplasia intestinal, para estadificar el riesgo de CG. El método más preciso para la atrofia es el OLGA, que exige 5 biopsias: 2 del cuerpo, 2 del antro y 1 de la incisura angularis, marcadas y enviadas en frascos separados. En Colombia, no se ha evaluado el rendimiento de OLGA en el estudio de la atrofia gástrica. Materiales y métodos: estudio de casos y controles. Los casos son pacientes en quienes se hizo el muestreo de biopsias para el OLGA. Los controles pacientes con menos de 5 biopsias gástricas, sin el muestreo del OLGA. Resultados: 1599 casos y 4191 controles. Edad promedio de los casos: $49 \pm 12$ años versus controles $54 \pm 10$ años (p: NS). H. pylori: $60 \%$ versus $57 \%$. GCA en casos: $42 \%$ versus $26 \%$. El $12,3 \%$ tenía OLGA III/IV y el $88 \%$, OLGA 0 , I o II, los cuales no ameritarían vigilancia endoscópica. Conclusión: el sistema OLGA permite detectar un $61,8 \%$ más de atrofia que la detectada con un muestreo insuficiente de biopsias gástricas. La mayoría de los casos (88\%) tuvo bajo riesgo de CG (estado 0-II) y no se justificaría vigilancia endoscópica.
\end{abstract}

\section{Palabras clave}

Gastritis atrófica, metaplasia intestinal, Helicobacter pylori, sistema Sídney, sistema OLGA.

\begin{abstract}
Introduction: Chronic atrophic gastritis (GCA) is a clinicopathological entity related to intestinal type gastric cancer (GC) whose main cause is helicobacter pylori. Currently, in addition to the diagnosis, it is recommended that the extent of atrophy or intestinal metaplasia be evaluated in order to stage the GC risk. The most accurate method for atrophy is OLGA which requires five biopsies: two from the corpus, two from the antrum and one from the angular incisure. Each biopsy is marked placed in a separate tube and marked. In Colombia, the use of OLGA to study gastric atrophy had not been evaluated previously. Materials and methods: This is a case and control study whose cases are patients who had biopsies taken to be studied with OLGA. Control patients had less than five gastric biopsies, without OLGA sampling. Results: This study includes 1,599 cases and 4,191 controls. The average age of cases was $49+/-12$ years, and the average age of controls was 54 $+/-10$ years (p: NS). H. pylori infections were found in $60 \%$ of the cases and in $57 \%$ of the controls while $42 \%$ of the cases were found to have gastric cancer and $26 \%$ of the cases were found to have GC. $12.3 \%$ had OLGA III or IV and $88 \%$ had OLGA 0, I or II and did not merit endoscopic monitoring. Conclusion: The OLGA system detects $61.8 \%$ more atrophy than is detected with less sampling of gastric biopsies. Most of the cases (88\%) had low risk of GC (stages $0-1 I)$ and did not require endoscopic monitoring.
\end{abstract}

\section{Keywords}

Atrophic gastritis, intestinal metaplasia, helicobacter pylori, Sidney system, OLGA system. 


\section{INTRODUCCIÓN}

La gastritis crónica (GC) es una entidad patológica muy frecuente en países con alta prevalencia y alta mortalidad por cáncer gástrico como el nuestro (1-3). Constituye un paso muy importante en la historia natural de la oncogénesis gástrica y su relación con el carcinoma gástrico de tipo intestinal está bien documentada (4). La atrofia de la mucosa gástrica y la metaplasia intestinal son consideradas condiciones precursoras de cáncer gástrico (CG) (5-10). En Holanda, se ha encontrado que la incidencia anual de CG en pacientes con atrofia es del 0,1\% y en metaplasia intestinal, del 0,25\% (11), y el riesgo de CG es del 2\%-3\% durante el seguimiento a diez años (11). Su adecuado diagnóstico mediante biopsias endoscópicas es fundamental para un correcto seguimiento de los pacientes (12). Las características histopatológicas de la gastritis crónica atrófica (GCA) son inflamación crónica y pérdida de las glándulas de la mucosa gástrica con atrofia o reemplazo de las mismas por epitelio de tipo intestinal (atrofia con metaplasia intestinal) (13-16). La presencia de glándulas antropilóricas en la mucosa corporal (metaplasia pseudopilórica), en su fase inicial, también hace parte de este proceso $(1,11-15)$. La GCA es un evento tardío en la evolución de la gastritis crónica, cuya principal causa es el Helicobacter pylori $(1-4,12)$, el cual es también la principal causa de CG $(4,7,12)$. Otro tipo menos frecuente de GCA es la gastritis autoinmune, que predomina en otras latitudes y es considerada muy rara en Colombia $(7,17,18)$. La GCA, asociada con $H$. pylori, es multifocal y compromete tanto la mucosa antral como la corporal, mientras que la gastritis atrófica autoinmune está restringida a la mucosa corporal $(1,13-16)$. La distribución de la alteración, en parches, motivó que Sídney (19) recomendara tomar 2 biopsias del cuerpo y 2 del antro para identificar adecuadamente dicha alteración. Sin embargo, en la actualización de este sistema, se consideró necesario adicionar una biopsia de la incisura angularis (20). Este mapeo fue recomendado por el último consenso de Maastricht (12). Otros autores han considerado que esa biopsia de la incisura es innecesaria, y en la reciente guía europea sobre el manejo de las lesiones precancerosas gástricas se ha respaldado tomar solamente biopsias del cuerpo y del antro sin incluir biopsia de la incisura (21). No obstante, la incisura es el sitio donde más tempranamente ocurren los cambios de atrofia y metaplasia en la GCA multifocal $(22,23)$. Este concepto fue tenido en cuenta por un grupo de gastroenterólogos y patólogos expertos que propusieron el sistema OLGA (Operative Link for Gastritis Assessment) como el instrumento para evaluar y determinar la extensión de la atrofia y de la metaplasia intestinal (24). Su puntuación va de 0 -IV, donde 0 es no atrofia, I, atrofia mínima, y IV, grado más severo de la misma (24). El propó- sito final del sistema OLGA es estratificar el riesgo de CG hacía el futuro $(12,24)$. Se recomienda que los pacientes que tengan OLGA III o IV sean vigilados con endoscopia para detectar precozmente el CG $(4,12,24)$. En un estudio de 93 pacientes con dispepsia, seguidos durante 12 años, se encontró que los únicos 2 pacientes que al final del seguimiento tuvieron CG habían sido clasificados como OLGA III/IV en la endoscopia inicial (25).

De manera similar al OLGA, más recientemente se ha propuesto el sistema OLGIM como un sistema para evaluar solamente los cambios de metaplasia intestinal (26). Aunque ambos sistemas de puntuación son útiles para estratificar el riesgo de CG e identificar a los pacientes que requieren seguimiento o vigilancia, el OLGIM tiene la ventaja de mayor concordancia interobservador; sin embargo, recientemente se encontró que puede subestadificar los casos que con el OLGA serían severos (III y IV) que ameritarían vigilancia ulterior (27).

Con los endoscopios de luz blanca, la correlación entre la apariencia de la mucosa y el grado de atrofia histológica es muy pobre, pero con los endoscopios de magnificación y alta resolución o endoscopios de imagen resaltada combinada con magnificación y cromoendoscopia electrónica (NBI), la correlación es muy alta en manos de endoscopistas bien entrenados en el uso de los mismos (28-33). Utilizando esta tecnología, las biopsias pueden ser dirigidas a los sitios de alteración morfológica de la mucosa y no tomar tantas biopsias al azar (30-32). Estos métodos avanzados son rutinariamente utilizados en Japón, pero todavía no están disponibles en el resto del mundo, incluida Colombia.

En nuestro país, hay alta prevalencia de H. pylori, de GCA y alta incidencia de CG, el cual es la principal causa de muerte por cáncer en hombres y la tercera causa de muerte en las mujeres (34). Por lo anterior, y por la falta de estudios en Colombia, decidimos realizar el presente trabajo con el fin de determinar si en nuestro medio, el adecuado muestreo de la mucosa gástrica y el uso del sistema OLGA pueden incrementar o no el diagnóstico de la GCA, comparado con biopsias sin el protocolo requerido para aplicar dicho sistema.

\section{MATERIALES Y MÉTODOS}

Estudio de casos y controles realizado en el Departamento de Patología de la Facultad de Medicina de la Universidad Nacional de Colombia (UN), Bogotá, utilizando la base de datos de los estudios histopatológicos realizados durante el período comprendido entre 2010 y 2013. El Departamento de Patología de la UN recibe muestras de pacientes enviados de todo Bogotá. Bogotá es una ciudad incluyente, que probablemente da la mejor representación del país. Estadísticas oficiales informan que cada año llegan a esta 
ciudad entre 400000 y 600000 personas. Por tanto, muchas regiones de Colombia están representadas en Bogotá.

Los casos fueron definidos como los pacientes cuyas biopsias gástricas se tomaron siguiendo el protocolo actualizado de Sídney, el cual permitió el uso del sistema OLGA: 2 muestras del antro ( 1 de la curva menor y 1 de la curva mayor tomadas a 3-4 cm del píloro), 2 biopsias del cuerpo ( 1 de la curvatura mayor de $8 \mathrm{~cm}$ del cardias y 1 de la curvatura menor de $4 \mathrm{~cm}$ proximal a la incisura) y 1 biopsia de la incisura angularis, todas enviadas en frascos separados, adecuadamente marcados $(20,24)$. Estas biopsias fueron tomadas durante endoscopias digestivas realizadas por gastroenterólogos o cirujanos endoscopistas. Una vez tomadas, fueron fijadas en formol al $10 \%$ y enviadas al Departamento de Patología. En el laboratorio, se siguió el proceso estándar de embeberlas en parafina y de cada bloque obtener múltiples secciones histológicas de 5 micras, las cuales fueron coloreadas con hematoxilina y eosina (HE) y adicionalmente coloración de Giemsa, cuando el $H$. pylori no fue detectado con HE (18). Todas las biopsias fueron evaluadas por patólogos expertos que generalmente disponían del informe endoscópico de los pacientes. Cuando había dudas, el informe final se decidió por el consenso entre los dos patólogos. Cada biopsia fue evaluada de acuerdo con el sistema actualizado de Sídney, y la graduación de la atrofia y la metaplasia en los diferentes fragmentos se realizó de acuerdo con las recomendaciones del sistema de puntaje de OLGA (24). El análisis de cada biopsia se hizo utilizando una lista de chequeo de la base de datos con los criterios diagnósticos de las diferentes categorías del sistema Sídney (20).

Los controles fueron los pacientes cuyas biopsias gástricas no fueron tomadas siguiendo el muestreo recomendado por el sistema actualizado de Sídney. El procesamiento de las biopsias gástricas fue igual al de las biopsias de los casos.

En ambos grupos se determinó la proporción de casos de GCA en relación con los otros tipos de gastritis, diagnósticos por grupos de edad, género y estado de infección por $H$. pylori. Se comparó la frecuencia de GCA en ambos grupos.

Los criterios de exclusión fueron los especímenes diferentes a biopsias gástricas (gastrectomías y polipectomías), los obtenidos como parte de biopsias de la unión gastroesofágica, los estudios de seguimiento histológico y las biopsias gástricas con diagnóstico de tumor. También se excluyeron los estudios de control de cualquier paciente.

\section{Análisis estadístico}

Todos los datos recolectados fueron procesados en Microsoft Office 2007 y analizados con el programa estadístico GraphPad StatMate 2. Para la comparación entre las proporciones y dicotómicas, se hizo Chi cuadrado y entre los promedios, con $\mathrm{t}$ de Student. Se consideró significancia estadística cuando la diferencia era menor al 0,05.

\section{RESULTADOS}

\section{Casos}

Durante el período de estudio, hubo 1599 casos estadificados y graduados mediante el sistema OLGA. En este grupo, 1028 (64\%) pacientes fueron mujeres y 571 (36\%) hombres, con promedio de edad de $49 \pm 10$ años (rango: 6-88).

La GCA se encontró en el $42 \%(n=665)$ de los pacientes y la no atrófica, en el 54\% ( $\mathrm{n}=869)$. En el 4\% (65 pacientes) se encontró gastropatía reactiva. Estas alteraciones histológicas se muestran en la figura 1.

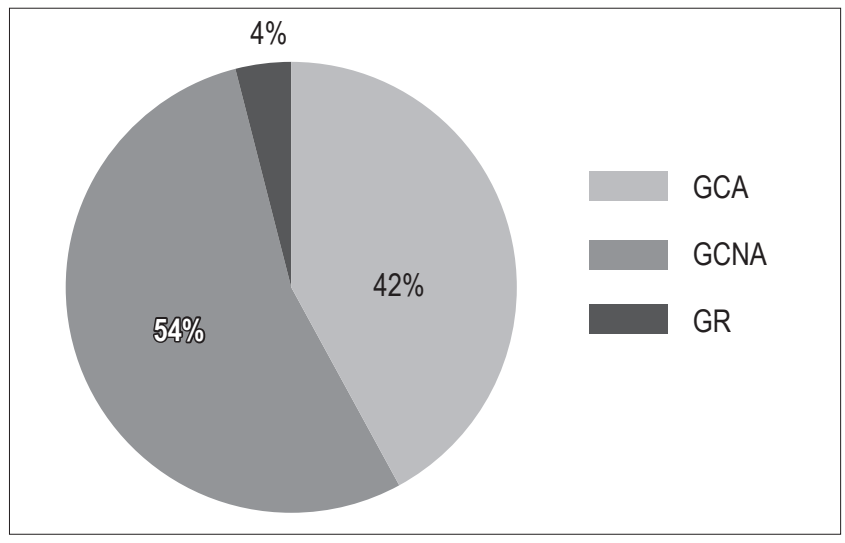

Figura 1. Alteraciones histológicas en los casos. GCA: gastritis crónica atrófica; GCNA: gastritis crónica no atrófica; GR: gastritis reactiva.

La distribución de la gastritis crónica atrófica multifocal (GCAMF) por grupos de edad se muestra en la tabla 1.

Tabla 1. Distribución por grupos de edad de las GCA del grupo de casos

\begin{tabular}{cc}
\hline Grupo & $\mathbf{n}(\%)$ \\
\hline $0-19$ & $3(0,5)$ \\
$20-29$ & $8(1)$ \\
$30-39$ & $37(5,5)$ \\
$40-49$ & $140(21)$ \\
$50-59$ & $198(30)$ \\
$60-69$ & $178(27)$ \\
$>70$ & $101(15)$ \\
\hline Total & $665(100)$ \\
\hline
\end{tabular}

Otras alteraciones concomitantes fueron gastropatía reactiva en 3 pacientes con GCAMF. Se reportaron 4 casos con gastritis atrófica corporal (autoinmune); la edad promedio de estos pacientes fue de 46 años, mientras que la edad de los que tenían GCAMF fue de 56 años $(\mathrm{p}<0,05)$. 
La distribución de los casos según el estado OLGA se muestra en la tabla 2.

Tabla 2. Distribución de los pacientes de acuerdo con el OLGA

\begin{tabular}{ccc}
\hline Estado de OLGA & $\mathbf{n}$ & $\%$ \\
\hline O & 934 & $54,3 \%$ \\
I & 300 & $19,8 \%$ \\
II & 176 & $11,6 \%$ \\
III & 162 & $10,6 \%$ \\
IV & 27 & $1,7 \%$ \\
\hline Total & 1599 & $100 \%$ \\
\hline
\end{tabular}

En el 3\% de los pacientes con GCA se encontró displasia $(\mathrm{n}=20)$. La frecuencia de displasia, de acuerdo con la categoría de OLGA, fue la siguiente: $70 \%$ ocurrió en los pacientes con OLGA III $(\mathrm{n}=14) ; 20 \%$, con OLGA IV ( $\mathrm{n}=$ $4)$; y $10 \%$, con OLGA $(\mathrm{n}=2)$ (figura 2$)$.

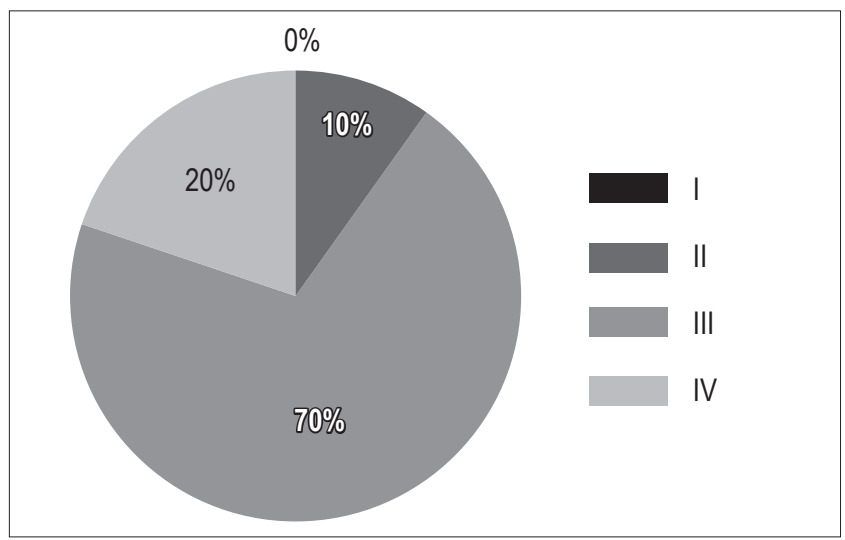

Figura 2. Displasia de acuerdo con el estadio de OLGA.

Los pacientes con OLGA 0 tienen significativamente una menor edad (44 años) que los de OLGA III y IV ( 59 y 65 años, respectivamente). La distribución de los estados del OLGA por grupo de edad se muestra en la tabla 3. En el 98,5\% de los pacientes con GCNA (OLGA 0) no había otras alteraciones y en el 1,5\% había además gastritis reactiva.
La infección por $H$. pylori se presentó en 400/665 casos (60\%) de GCA sola o asociada con otro diagnóstico, en $633 / 869$ casos (73\%) de GCNA (OLGA 0) sola o asociada con otro diagnóstico $(\mathrm{p}<0,05)$ y estuvo ausente en los 65 casos de gastropatía reactiva.

\section{Controles}

Durante el período de estudio hubo 4191 controles. De estos, 2553 (61\%) fueron mujeres. La edad promedio de todo el grupo fue de 54 años (rango 10-98). Se encontró GCAMF en el 26\% (1113) de los pacientes, GCNA en el $68 \%$ y gastropatía reactiva en el $6 \%$. Estas características histológicas se muestran en la figura 3. La infección por $H$. pylori se presentó en el 58\% (2443) de los pacientes.

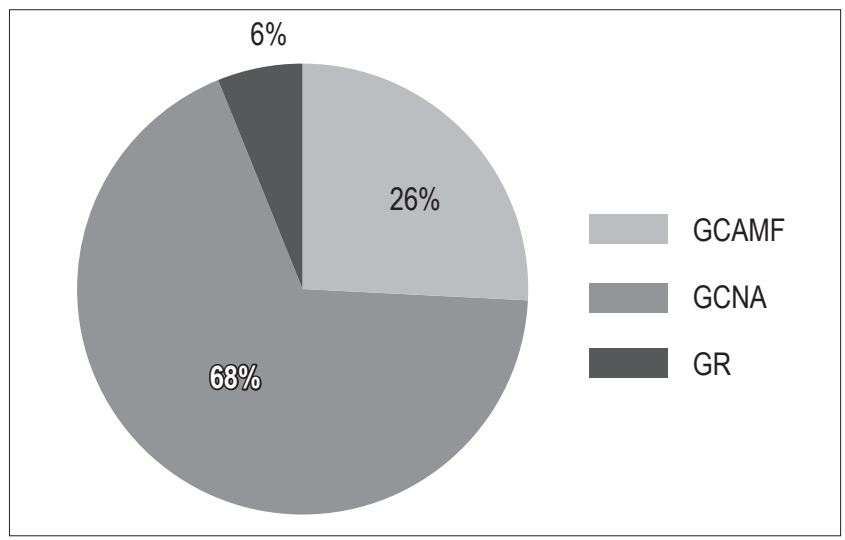

Figura 3. Hallazgos histológicos en el grupo control. GCAMF: gastritis crónica atrófica multifocal; GCNA: gastritis crónica no atrófica; GR: gastritis reactiva.

De los pacientes con GCA, 42\% fueron hombres (471). $\mathrm{El}$ promedio de edad fue 61 años (rango 50-80 años). Se presentó como diagnóstico único en 946/1113 casos (85\%) y asociada con GR en 167/1113 casos (15\%). La GCNA correspondió al 68\% del total de casos $(\mathrm{n}=2836)$. Se presentó como diagnóstico único en 2585 casos (91\%) y en 251 (9\%) se asoció con GR. Esta última se encontró

Tabla 3. Estados del OLGA en relación con la edad en el grupo de casos

\begin{tabular}{lccccccccc}
\hline Estadio & $\mathrm{n}$ & Promedio de & \multicolumn{7}{c}{ Subgrupos de edad por estados $\mathrm{n}(\%)$} \\
\cline { 6 - 10 } OLGA & $(\%)$ & edad (rango) & $\mathbf{0 - 1 9}$ & $\mathbf{2 0 - 2 9}$ & $\mathbf{3 0 - 3 9}$ & $\mathbf{4 0 - 4 9}$ & $\mathbf{5 0 - 5 9}$ & $\mathbf{6 0 - 6 9}$ & $\mathbf{7 0}$ \\
\hline 0 & $933(58,4)$ & $44(6-88)$ & $33(92)$ & $136(94,4)$ & $204(84,6)$ & $220(61,1)$ & $200(50,2)$ & $95(34,8)$ & $46(31,3)$ \\
I & $301(18,8)$ & $54(16-82)$ & $1(3)$ & $6(4,2)$ & $26(10,8)$ & $73(20,3)$ & $87(21,9)$ & $80(29,3)$ & $27(18,4)$ \\
II & $176(11)$ & $57(18-84)$ & $2(5)$ & $1(0,7)$ & $5(2,1)$ & $40(11,1)$ & $56(14,1)$ & $46(16,8)$ & $29(19,7)$ \\
III & $162(10,1)$ & $59(26-87)$ & 0 & $1(0,7)$ & $6(2,5)$ & $24(6,7)$ & $49(12,3)$ & $45(16,5)$ & $34(23,1)$ \\
IV & $27(17)$ & $65(43-83)$ & 0 & 0 & 0 & $3(0,8)$ & $6(1,5)$ & $7(2,6)$ & $11(7,5)$ \\
\hline Total & $1599(100)$ & & $36(100)$ & $144(100)$ & $241(100)$ & $360(100)$ & $398(100)$ & $273(100)$ & $147(100)$ \\
\hline
\end{tabular}


como diagnóstico único en el 6\% de los casos (242/4191). En la tabla 4 se muestra la comparación entre los casos y los controles, con respecto a las principales características.

Tabla 4. Comparación entre los casos y los controles

\begin{tabular}{lccc}
\hline & $\begin{array}{c}\text { Casos } \\
(\mathbf{n}=\mathbf{1 5 9 9})\end{array}$ & $\begin{array}{c}\text { Controles } \\
(\mathbf{n}=\mathbf{4 1 9 1 )}\end{array}$ & $\mathbf{p}$ \\
\hline $\begin{array}{l}\text { Gastritis crónica atrófica } \\
\text { Gastritis crónica no atrófica }\end{array}$ & $42 \%(665)$ & $26 \%(1113)$ & $<0,001$ \\
Gastropatía reactiva & $4 \%(65)$ & $6 \%(242)$ & NS \\
H. pylori & $60 \%(1033)$ & $57 \%(2443)$ & NS \\
\hline
\end{tabular}

\section{DISCUSIÓN}

En el presente estudio de casos y controles, con una población superior a los 5700 pacientes, se comparó la frecuencia de atrofia cuando se utilizó el muestreo recomendado por el sistema actualizado de Sídney versus un muestreo que no siguió este protocolo. En los casos se aplicó el sistema OLGA para graduar y estadificar la atrofia, y se encontró gastritis crónica atrófica multifocal en el $42 \%$ de los pacientes versus el $26 \%$ del grupo control ( $\mathrm{p}<0,001)$, lo cual significa un rendimiento del $61,8 \%$ más con el muestreo de 5 biopsias. Además de un mayor número de biopsias en el cuerpo y el antro, una diferencia clave entre estos dos grupos fue la biopsia de la incisura, cuyo impacto se ha demostrado $(35,36)$. Recientemente un estudio de Lituania encontró que cuando no se incluye la biopsia de la incisura, el grado de severidad de la atrofia y de la metaplasia intestinal, mediante el sistema OLGA y OLGIM, respectivamente, disminuye en $18 \%$ y $4 \%$, respectivamente, y cuando se evalúan estados de alto riesgo (grado III y IV), se deja de detectar el $30 \%$ y el $35 \%$, de casos respectivamente (35). En ese estudio (35) y en otro (36), se demostró que la incisura es más confiable que el cuerpo y el antro para detectar atrofia y metaplasia intestinal. En el presente estudio por definición no se pudo estimar el OLGA en los controles, pero la GCA se encontró significativamente de manera más frecuente en los casos. Esta mayor diferencia, comparada con lo encontrado en el trabajo lituano, probablemente se deba a que en el grupo control también se incluyeron pacientes con solo 1 o 2 biopsias, lo que limita aún más la identificación de la atrofia. La importancia de un muestreo gástrico completo que incluya las 5 biopsias, y biopsias adicionales si hay lesiones específicas, fue tenida en cuenta por el último consenso de Maastricht (12) y más recientemente por el consenso Global de Kioto sobre gastritis crónica y $H$. pylori (37).

Para atribuir el mayor rendimiento del sistema OLGA comparado con la práctica de tomar un número limitado de biopsias, para la detección de atrofia, es necesario descartar que en los casos no existieran otras variables que explicaran esa gran diferencia. Entre esas se encuentran fundamentalmente la edad y la prevalencia de $H$. pylori. Con respecto a la edad, los casos eran más jóvenes y teóricamente deberían tener menos atrofia; por tanto, la diferencia más probablemente se debió a la forma de tomar las biopsias. Con respecto al $H$. pylori, la prevalencia de la infección fue similar en ambos grupos, $60 \%$ y $57 \%$, respectivamente (p: NS), y prácticamente igual a la encontrada en un estudio realizado recientemente en Bogotá (38).

Aunque la biopsia gástrica constituye el estándar de oro para la evaluación y el seguimiento de la gastritis crónica, el muestreo usualmente utilizado en nuestro medio, como se encontró en este trabajo, no sigue el protocolo de Sídney, siendo inadecuado para el diagnóstico y graduación de la atrofia, que es la alteración más importante en la cadena de la carcinogénesis $(1-3,12,38)$. Colombia es un país clasificado como de alta incidencia de CG (34) y llama la atención que en este estudio, con el protocolo del sistema OLGA, se observó que solo el 12,3\% de los pacientes tenía alto riesgo de CG (OLGA III y IV) y ameritarían vigilancia endoscópica después de erradicarles $H$. pylori $(12,37)$. La inmensa mayoría $(88,7 \%)$ tenía mínimo riesgo (OLGA 0, I y II), en quienes teóricamente no sería necesaria la vigilancia endoscópica después de erradicarles $H$. pylori $(4,12)$. Consideramos que estos hallazgo, podrían impactar la rutina de las endoscopias digestivas en nuestro medio, ya que con un adecuado muestreo de biopsias se tendría la información para decidir la necesidad o no de vigilar a los pacientes con endoscopias hacia el futuro. Si solo el 12,3\% necesitan endoscopias de control, se evitarían gastos por la repetición de endoscopias innecesarias por gastritis en la mayoría de los pacientes.

De los 20 pacientes con displasia, 18 de ellos (90\%) tenían categorías de alto riesgo del sistema OLGA (III y IV) y 2 (10\%), con estadio OLGA II, que representa el 1,13\% de estos pacientes. El hallazgo de que la mayoría de casos con displasia (80\%) se encontrara en pacientes con OLGA III/IV coincide con los resultados de un estudio Japonés de Satoh y colaboradores (39). En dicho estudio se encontró que el 84\%\% de los pacientes con CG tenía OLGA III/IV, lo cual contrasta con los pacientes con úlcera duodenal, la mayoría de los cuales tenía OLGA 0/I y la minoría, OLGA III/IV. Hallazgos similares también han sido encontrados por Rugge y colaboradores (40). En ese estudio, 7 pacientes con CG estaban entre los 21/439 pacientes con OLGA III/IV (40).

El hallazgo de displasia en 2/176 pacientes con OLGA II ( $1,36 \%)$, ameritaría más estudios, ya que dicho grupo de pacientes tradicionalmente no se considera de riesgo para vigilarlos de manera prospectiva con endoscopia digestiva alta (4). Si se corroboran estos resultados, se podría comenzar a discutir la necesidad de vigilar también a estos pacientes. 
Los casos con estados III y IV de OLGA predominaron en pacientes de mayor edad con respecto a los estados I y II, lo cual puede indicar que aquellos han tenido un proceso de inflamación crónica más largo que los estadios más leves $(4,12,23)$. El sistema OLGA, además de permitir seleccionar pacientes con mayor riesgo de desarrollo de cáncer gástrico con base en la extensión de los cambios de atrofia y metaplasia intestinal (estados III y IV), permite identificar la aparición de estos cambios en individuos jóvenes en fases tempranas, en quienes el proceso puede evolucionar en décadas ulteriores a estados avanzados si no hay una intervención que permita detener su progresión $(4,12)$. El riesgo de CG en pacientes con OLGA IV es 10-90 veces (41) y depende de la extensión de la atrofia y no de la edad, pues esta última no es un factor de riesgo independiente para el CG (41). En nuestro país no hay datos exactos sobre la probabilidad de CG en pacientes con atrofia leve o extensa y consideramos que esto ameritaría estudios de seguimiento en esas cohortes de pacientes.

Dada la importancia de la vigilancia para detectar precozmente un CG, consideramos que a los pacientes con OLGA III/IV que se les haga seguimiento, la endoscopia debe ser detallada y con el protocolo de detección de CG temprano (42). Sería injustificado llevar a cabo una endoscopia de rutina y repetir el muestreo subóptimo de biopsias.

En este estudio, el 99\% de los casos de GCA fue multifocal; solo el $1 \%$ fue corporal aislado, que es el patrón de las GCA autoinmunes, y se encontró solamente en los casos que por definición tuvieron el muestreo de las 5 biopsias (24). Tomando en conjunto la población de casos, estos 4 pacientes solo representan el $0,25 \%$ de toda la muestra, lo que corrobora el concepto de que esta gastritis es muy rara en nuestro medio $(1,2)$.

El presente es el primer estudio realizado en Colombia que analiza el impacto del sistema OLGA en la estratificación de riesgo de los pacientes con gastritis crónica. Somos conscientes de las limitaciones de este trabajo. Es un estudio retrospectivo; no se tuvo la información clínica sobre los antecedentes del paciente, tales como estrato socioeconómico, antecedentes familiares de CG ni terapias de erradicación de $H$. pylori. Sin embargo, con respecto a esta última limitación, la prevalencia de $H$. pylori fue igual a la encontrada en un estudio nacional que tenía entre sus objetivos determinar la prevalencia de la infección en personas adultas sin antecedente de terapias de erradicación.

\section{CONCLUSIONES}

- En el presente estudio, con una muestra de más de 5000 pacientes, se ha demostrado que con el protocolo de
OLGA se identificó un $61,8 \%$ más de casos de atrofia que utilizando protocolos con menos biopsias ( $42 \%$ versus $26 \%$ ).

- Es el primer estudio en nuestro país que demuestra la asociación de displasia con estadios avanzados de OLGA (III/IV), similar a los encontrados en estudios de otros países con alta incidencia de cáncer gástrico (CG), como Japón.

- Un hallazgos sorprendente y que podría ser tenido en cuenta en los programas de identificación precoz del CG es que la mayoría de los casos de GC (88\%) no tenía riesgo de CG (OLGA 0-II). Este hallazgo implicaría que la mayoría de personas no tendría necesidad de ser sometida a endoscopias de control o vigilancia. Sin embargo, sería importante que en cada región de Colombia se realicen estudios, ya que dependiendo de los resultados, las políticas de salud podrían cambiar, porque la epidemiología del CG puede variar, incluso dentro de un mismo país (34).

- La gastritis autoinmune es prácticamente inexistente en nuestro país (4/1599) (0,4\%).

- En un país como Colombia, con alta incidencia de CG, es urgente que la comunidad de gastroenterólogos y endoscopistas incorpore en su práctica el adecuado protocolo de las 5 biopsias gástricas, enviadas por separado ( 2 del cuerpo, 2 del antro y 1 de la incisura), ya que cuando no se realiza de esta manera, hay un subdiagnóstico de la atrofia. La biopsia de la incisura es definitiva. En este trabajo se ha evaluado el OLGA, pero también se puede utilizar OLGIM de acuerdo con los más recientes consensos internacionales.

- Nuestros resultados podrían impactar a nivel nacional la rutina de las biopsias gástricas durante las endoscopias. Consideramos que como una política de salud pública, el Estado y las empresas de salud deben garantizar el costo que implica esta forma correcta de tomar las biopsias del estómago.

- Con el protocolo de OLGA se podrían disminuir las endoscopias de control por gastritis, en las cuales nuevamente se toman biopsias insuficientes. En este trabajo se ha observado que la mayoría de los pacientes (>85\%), teóricamente no ameritarían endoscopias de vigilancia. Ese ahorro permitiría los recursos para hacer un adecuado muestreo, con las biopsias en la endoscopia inicial, de acuerdo con el estándar actual.

\section{Conflictos de intereses}

Ninguno. Los costos del presente estudio fueron asumidos por los investigadores. 


\section{REFERENCIAS}

1. Correa P, Cuello C, Duque E, et al. Gastric cancer in Colombia. III. Natural history of precursor lesions. J Natl Cancer Inst. 1976;57:1027-35.

2. Correa P, Piazuelo MB, Wilson KT. Pathology of gastric intestinal metaplasia: clinical implications. Am J Gastroenterol. 2010;105:493-8.

3. Correa P, Fontham ET, Bravo JC, et al. Chemoprevention of gastric dysplasia: randomized trial of antioxidant supplements and anti-Helicobacter pylori therapy. J Natl Cancer Inst. 2000;92:1881-8.

4. Graham DY. Helicobacter pylori update: gastric cancer, reliable therapy and possible benefits. Gastroenterology. 2015;148:719-31.

5. Kuipers EJ. Review article: Relationship between Helicobacter pylori, atrophic gastritis and gastric cancer. Aliment Pharmacol Ther. 1998;(12)Suppl 1:25-36.

6. Correa P. Human gastric carcinogenesis: a multistep and multifactorial process - a first American Cancer Society Award Lecture on Cancer Epidemiology and Prevention. Cancer Res. 1992;52(24):6735-40.

7. Sipponen P, Maaroos HI. Chronic gastritis. Scand J Gastroenterol. 2015;50(6):657-67.

8. Correa P, Piazuelo MB. Natural history of Helicobacter pylori infection. Dig Liver Dis. 2008;40(7):490-6.

9. Atherton JC. The pathogenesis of Helicobacter pyloriinduced gastro-duodenal diseases. Annu Rev Pathol. 2006; (1):63-96.

10. Weck MN, Gao L, Brenner H. Helicobacter pylori infection and chronic atrophic gastritis: associations according to severity of disease. Epidemiology. 2009;20(4):569-74.

11. De Vries AC, van Grieken NC, Looman CW, et al. Gastric cancer risk in patients with premalignant gastric lesions: a nationwide cohort study in the Netherlands. Gastroenterology. 2008; 134:945-52.

12. Malfertheiner P, Megraud F, O'Morain CA, et al. Management of Helicobacter pylori infection-the Maastricht IV/Florence Consensus Report. Gut. 2012;61:646-64.

13. Rugge M, Pennelli G, Pilozzi E, et al. Gastritis: the histology report. Dig Liver Dis. 2011; (43)Suppl 4:S373-84.

14. Lewin D, Lewin K. Stomach. En: Weidner N, Cote RJ, Suster S, et al (editores). Modern surgical pathology. 2. ${ }^{\text {a }}$ edición. Canada: Expert Consult; 2009. p. 703-10.

15. Fenoglio-Preiser C. Creating a framework for diagnosing the benign gastric biopsy. Curr Diagn Pathol. 1998;5:2-16.

16. El-Zimaity HM. Recent advances in the histopathology of gastritis. Current Diag Pathol. 2007;13:340-8.

17. Correa P. Chronic gastritis: a clinic-pathological classification. Am J Gastroenterol. 1998;83(5):504-9.

18. Ricaurte O, Gutiérrez O. Gastritis crónica. En: Alvarado J, Otero W, Archila PE, et al. Gastroenterología y hepatología. 2. a edición. Bogotá: Editorial Médica Celsus; Sociedad Colombiana de Endoscopia Digestiva; 2006. p. 549-64.

19. Price AB. The Sydney System: histological division. J Gastroenterol Hepatol. 1991;6:209-622.
20. Dixon MF, Genta RM, Yardley HJ, et al. Classification and grading of gastritis. The updated Sydney System. Am J Surg Pathol. 1996;20:1161-81.

21. Dinis-Ribeiro M, Arela M, de Vries AC, et al. Management of precancerous conditions and lesions in the stomach (MAPS): guideline from the European Society of Gastrointestinal Endoscopy (ESGE), European Helicobacter Study Group (EHSG), European Society of Pathology (ESP), and the Sociedade Portuguesa de Endodcopia Digestiva (SPED). Endoscopy. 2012;44:74-94.

22. Sugimura T, Sugano H, Terada M, et al. First International Workshop of the Princess Takamatsu Cancer Research Fund: intestinal metaplasia and gastric cancer. Mol Carcinog. 1994; 11(1):1-7.

23. Rugge M, Cassaro M, Pennelli G, et al. Atrophic gastritis: pathology and endoscopy in the reversibility assessment. Gut. 2003;52:1387-88.

24. Rugge M, Correa P, Di Mario F, et al. OLGA staging for gastritis: a tutorial. Dig Liver Dis. 2008;40(8):650-8.

25. Rugge M, de Boni M, Pennelli G, et al. Gastritis OLGAStaging and gastric cancer risk: a twelve-year clinicopathological follow-up study. Aliment Pharmacol Ther. 2010;31:1104-11.

26. Capelle LG, de Vries AC, Haringsma J, et al. The staging of gastritis with the OLGA systems by using intestinal metaplasia as an accurate alternative for atrophic gastritis. Gastrintest Endosc. 2010;71(7):1150-8.

27. Marcos-Pinto R, Carneiro F, Dinis-Ribeiro M, et al. Firstdegree relatives of patients with early-onset gastric carcinoma show even at young ages a high prevalence of advanced OLGA/OLGIM stages and dysplasia. Aliment Pharmacol Ther. 2012;35:1451-9.

28. Anagnostopoulus GK, Yao K, Kaye P, et al. High-resolution magnification endoscopy can reliably identify normal gastric mucosa. Helicobacter pylori-associated gastritis and gastric atrophy. Endoscopy. 2007;39(3):202-7.

29. Pimentel-Nunes P, Dinis-Ribeiro M, Soares JB, et al. Multicenter validation of an endoscopic classification with narrow band imaging for gastric precancerous and cancerous lesions. Endoscopy. 2012;44(3):236-46.

30. Kikuste I, Stirna D, Liepniece-Karele, et al. The accrue of flexible spectral imaging color enhancement for the diagnosis of gastric intestinal metaplasia: do we still need histology to select individuals at risk of adenocarcinoma? Eur J Hastroenterol Hepatol. 2014;26:704-10.

31. Uedo $\mathrm{N}$, Ishihara $\mathrm{R}$, Iishi $\mathrm{H}$, et al. A new method of diagnosing gastric intestinal metaplasia: narrow-band imaging with magnifying endoscopy. Endoscopy. 2006;38:819-24.

32. Tahara T, Shibata T, Nakamura M, et al. Gastric mucosal pattern by using magnifying narrow-band imaging endoscopy clearly distinguishes histological and serological severity of chronic gastritis. Gastrointest Endosc. 2009;70:246-53.

33. Kato M, Kaise M, Yonezawa J, et al. Magnifying endoscopy with narrow-band imaging achieves superior accuracy in the differential diagnosis of superficial gastric lesions iden- 
tified with white-light endoscopy: a prospective study. Gastrointest Endosc. 2010;72:523-9.

34. World Health Organization (WHO). GLOBOCAN 2012: Estimated Incidence, Mortality and Prevalence Worldwide in 2012. Disponible en: http://globocan.iarc.fr/.

35. Isajevs S, Liepniece-Karele I, Janciauskas D, et al. The effect of incisura angularis biopsy sampling on the assessment of the gastritis stage. Eur J Gastroenterol Hepatol. 2014;26:510-3.

36. Zhang C, Yamada N, Wu YL, et al. Helicobacter pylori infection, glandular atrophy and intestinal metaplasia in superficial gastritis, gastric erosion, erosive gastritis, gastric ulcer and early gastric cancer. World J Gastroenterol. 2005; 11:791-6.

37. Sugano K, Tack J, Kuipers EJ, et al. Kyoto global consensus report on Helicobacter pylori gastritis. Gut. 2015;64(9):1353-67.
38. Otero W, Trespalacios AA, Mercado M. Tasa de reinfección por Helicobacter pylori en una cohorte de pacientes colombianos tratados exitosamente con seguimiento superior a 2 años. Rev Col Gastroenterol. 2015;30:53-9.

39. Satoh K, Osawa H, Yoshizaewa M, et al. Assessment of atrophic gastritis using the OLGA system. Helicobacter. 2008; 13(3):225-9.

40. Rugge M, Meggio A, Pennelli G, et al. Gastritis staging in clinical practice: the OLGA staging system. Gut. 2007;56:631-6.

41. Sipponen P, Graham DY. Importance of atrophic gastritis in diagnostics and prevention of gastric cancer: application of plasma biomarkers. Scand J Gastroenterol. 2007;42:2-10.

42. Yada T, Yokoi Y, Uemura N. The current state of diagnosis and treatment for early gastric cancer. Diagn Ther Endosc. 2013;2013:241320. 